\title{
Frequency Response Estimation Method for Modelica Model and Frequency Estimation Toolbox Implementation
}

\author{
Bingrui Bao ${ }^{1}$ Junfeng Guo $^{1} \quad$ Baokun Zhang $^{1} \quad$ Fanli Zhou $^{1}$ \\ ${ }^{1}$ Suzhou Tongyuan Software\&Control Tech. Co., Ltd. China, \{baobr, guojf, zhangbk, zhoufl\} @ tongyuan. cc
}

\begin{abstract}
The frequency-response method is widely used because of its convenience and applicability in the control system analysis and design, and the premise of the design based on this method is to obtain the frequency response of the system. Aiming at the problem that the strong nonlinearity is difficult to be linearized in practical engineering systems, this paper presents a method for estimating the frequency response based on the time domain simulation data of Modelica models. The spectrum of the appropriate IO data at the steadystate operating point of the system is analyzed by Fourier transform, and then converted into the frequency response of the system. The proposed method is applicable to the multi-domain Modelica model, FMU, and the black box model. A frequency response estimation toolbox is implemented and integrated into the MWorks platform based on this method, which provides important support for the control design for Modelica model. Simulation examples illustrated the validity of the proposed method and the toolbox.
\end{abstract}

Keywords: Strong nonlinear system, Frequency response estimation, Toolbox

\section{Introduction}

Compared with time domain analysis, the frequency domain analysis can provide more intrinsic properties of the system. The design of feedback control systems in industry is probably accomplished using frequencyresponse methods more often than any other (Dorf et al, 2016; Ogata, 2010). The frequency-response design is popular primarily because it provides good design ability in the case of uncertainty in the plant which is difficult to describe with accurate mathematical model. On the other hand, it is the easiest method to use for designing compensation (Franklin et al, 2009). The premise of feedback control design based on frequency response method is to obtain the frequency response of the system.

In Brief, the theoretical analysis and experimental estimation are two fundamentally methods for obtaining system frequency-response. As regards the theoretical analysis method, which performs Laplace transform on the mathematical model of the system to obtain its transfer function, and then replaces the Laplace operator $\boldsymbol{s}$ with $\boldsymbol{j} \boldsymbol{\omega}$ to obtain the frequency response model. Speaking of the experimental method, it could be introduced as estimating system frequencyresponse by means of deeming system to be black box and then processing time-domain IO signal which is obtained from experiments.

The plant of industry systems are generally characterized by multi-domain, high stiffness and strong nonlinearity, thus Modelica has its natural advantages in describing systems with these characteristics (Fritzson, 2010). Meanwhile, plenty of remarkable studies on method of frequency-domain analysis of Modelica model have been conducted all this time. Martin Otter introduced the LinearSystems library in 2006, the library provides a definition of linear systems and also can be used to analyze it, including analysis of frequency response for linear systems (Otter, 2006). Andreas Abel et al. proposed a frequency domain analysis method for Modelica model based on the periodic steady-state simulation by using SimulationX, the transfer function and the frequency domain related properties of the system can be obtained by the models linearized in an operating point (Abel, 2008). Loig Allain et al. performed the linear analysis methods by the existing facilities in LMS Imagine.Lab, which is essentially a linearization method based on small perturbation theory (Allain et al, 2009). The LinearSystems 2 library is an updated version of the LinearSystems library was completed in 2009 by Marcus Baur, Martin Otter, etc., which enhances and extends the functionality of the original library (Baur et al, 2009), for example, a linearize function for linearization of physical models is added. Tilman Bünte proposed a frequency response estimation method based on time-domain data (Bünte, 2011). However, for strongly nonlinear systems, timedomain data acquired from Chirp signals have poor performance in estimation. Based on the linearization method provided by Dymola, Garron Fish et al. analyzed the natural frequencies of powertrain systems, the linearization function of Dymola is also based on the small perturbation theory (Fish et al, 2012).

The research above of frequency-domain analysis for Modelica model can be divided into two categories: frequency-analysis based on linearization and 
frequency-response estimation based on IO data from model. The shortcomings of the current researches can be summarized as follows.

- For one, Linearization is generally based on the small perturbation theory, which have to meet two requirements. Firstly, there should be at least one steady-state operating point in system. Secondly, all-order derivatives of equations describing the system at the operating point require to be existed. It cannot be stressed enough that the second requirement could be hardly met.

- For another, neither user-friendly process of existing data-based frequency response estimation has been standardized nor has method of obtaining time-domain data with acceptable estimation results been put forward.

- In addition, user-friendly time-domain data based frequency response estimation toolbox has not been formed yet.

Given the difficulties of frequency analysis on Modelica model, the topics of study on obtaining the appropriate time-domain data for estimating system frequency response of a class of SIMO(single input multiple output) Modelica model are as follows.

- A general process and key algorithm of data based frequency response estimation for Modelica model.

- A time-domain data obtainment method suitable for estimating the frequency response of strongly nonlinear system.

- Frequency response estimation toolbox which is developed on the basis of above method.

\section{Frequency Response Estimation Method for Modelica Model}

Linear system response to sinusoidal inputs is named as the system frequency response. Frequency of the input signal is varied in a certain range for studying the resulting response in the method of frequency response estimation. The following state space models are used to represent the general continuous-time nonlinear Modelica model for the convenience of explaining the problem.

$$
\begin{aligned}
& \dot{\boldsymbol{x}}(t)=f[\boldsymbol{x}(t), \boldsymbol{u}(t), t] \\
& \boldsymbol{y}(t)=g[\boldsymbol{x}(t), \boldsymbol{u}(t), t]
\end{aligned}
$$

In models above, $t$ is the time and $\boldsymbol{x}(t)$ is the state variables of the system. Besides, $\boldsymbol{u}(t)$ is the input of the system and $\boldsymbol{y}(t)$ is the output. The frequency response estimation for the system described in equation (1) is performed near a certain steady-state operating point of the system. It is assumed that the system has a steady-state operating point $\boldsymbol{x}\left(t_{o p}\right)$ under the input $\boldsymbol{u}(t)$. As a result, the steady-state output of the system at the operating point can be expressed as $\boldsymbol{y}\left(t_{o p}\right)=g\left[\boldsymbol{x}\left(t_{o p}\right), \boldsymbol{u}\left(t_{o p}\right), t_{o p}\right]$.

\subsection{Principle of Frequency Response Estimation}

The designed small disturbance input signal $\Delta(t)$ is added to the existing input signal $\boldsymbol{u}(t)$ at the linearization input point in the method of frequency response estimation. A new input signal excitation system model for obtaining the output at the linearization output point is described as follows. $\boldsymbol{u}_{\text {test }}(t)=\boldsymbol{u}(t)+\Delta(t)$

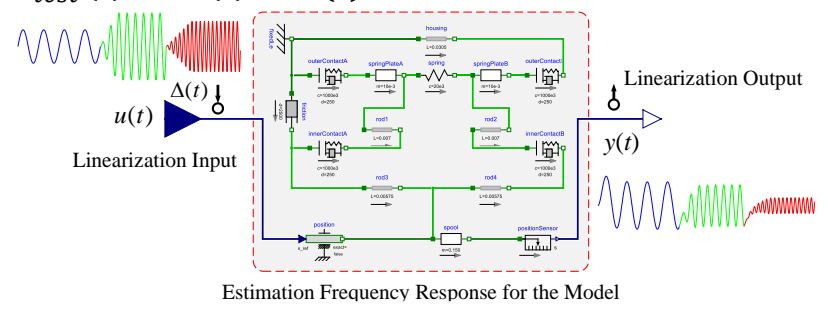

Figure 1. Acquisition of time domain data

There is a transient portion and steady state portion at each frequency of the simulated output $\boldsymbol{y}(t)$. The steady state portion is selected for sampling and filtering, etc. FFT transform is applied to the processed output signal to estimate the spectrum. Frequency response near the operating point can be easily calculated based on the spectrum of output signal.

\subsection{Frequency Response Estimation Algorithm}

\section{- Model encapsulation}

The model to be analyzed is encapsulated as a separate model. As is shown in Figure 1, linearized input point and linearized output point is defined. The following test signal will be added into the linearized input point. In consideration of the convenience of adding the test signal into input point, the type of the input interface is set as RealInput.

\section{- Create Estimation Input Signals}

Chirp signal and Sinestream signal are commonly used for frequency response estimation as disturbance signals.

$$
\begin{gathered}
\operatorname{chirp}(\omega, t)=A \cdot \sin (\omega t) \\
t_{s} \leq t \leq t_{e}, \omega_{s} \leq \omega \leq \omega_{e} \\
\operatorname{sinestream}(t)=\left\{\begin{array}{c}
A_{0} \cdot \sin \left(\omega_{0} t\right), t_{s} \leq t<t_{0} \\
A_{1} \cdot \sin \left(\omega_{1} t\right), t_{0} \leq t<t_{1} \\
\vdots \\
A_{n} \cdot \sin \left(\omega_{n} t\right), t_{n-1} \leq t<t_{n}
\end{array}\right.
\end{gathered}
$$

As is shown in equations (2) and (3), The Chirp signal and Sinestream signal are respectively expressed in equations (2) and (3), in which it can be seen that Chirp signal is a continuous signal while Sinestream signal is a segmentation signal. Chirp signal is suitable for system which is nearly linear in simulation range. However, if there existed strong nonlinearity in system and high accuracy of frequency response was required, 
Sinestream signal would be an excellent choice as disturbance signal.

Sinestream signal chosen as excitation signal is applied for time-domain data acquisition of system. To begin with, the range of frequency estimation along with number of the estimated points are defined appropriately. Secondly, steady-state operating point of the system needs to be set properly as well. Thirdly, amplitude, period and number of sampling points should be customized as attributes of each corresponding sinusoidal signal with specific frequency. The definitions of signal attributes are shown in Figure 2.

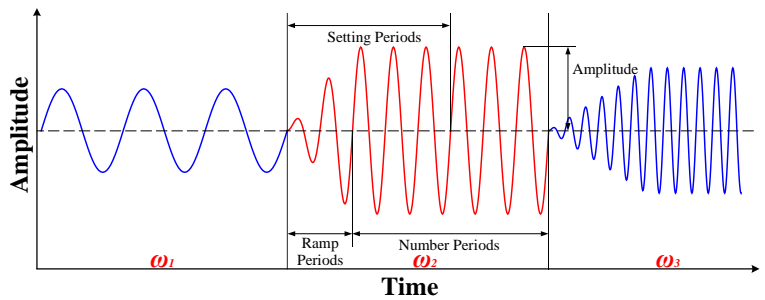

Figure 2. Definition of sine-stream signal attribute

According to the definition of the Sinestream signal above, model based on Modelica language can be easily implemented. Code part is omitted for lack of space.

\section{- Time Domain Simulation}

A Sinestream signal consists several adjacent sine waves with varying frequencies. Each frequency excites the system for a suitable time to ensure that the system could enter steady state. The output at the linearized output point is set as the original signal for the following estimation.

\section{- Signal Processing}

In order to ensure the accuracy of the estimation results, the output signal is divided into $n$ segments according to the duration of different frequency components. $n$ represents the number of different frequency points contained in the set frequency range. Figure 3 shows a schematic diagram of the system input signal and output signal at a specific frequency.

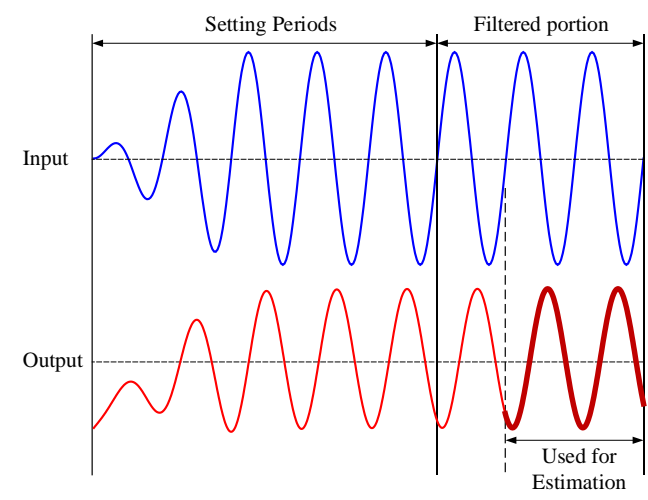

Figure 3. Signal selection

The simulated output at each frequency has a transient portion and steady state portion. Settling
Periods corresponds to the transient components of the output and input signals. The periods following Settling Periods are considered to be at steady state. Discards the Settling Periods portion of the output (and the corresponding input) at each frequency. Sampling the signal used for estimation, assuming that $F_{S}$ is the sampling frequency and $N$ is the number of sampling points.Two points need to be noted are as follows.

i. According to the sampling theorem, it is necessary to ensure that $F_{S}$ is two times larger than the signal frequency.

ii. In order to ensure the calculation speed of FFT transform, the number of sampling points $N$ is usually an integer power of two.

- Frequency Response Estimation

The discrete Fourier transform (DFT) is performed on the processed output data. $\boldsymbol{x}[n]$ is assumed to be the processed output at a certain frequency of which length is $n$. The DFT is performed as follows.

$$
\boldsymbol{Y}(k)=\sum_{j=1}^{n} \boldsymbol{x}(j) W_{n}^{(j-1)(k-1)}, W_{n}=e^{(-2 \pi i) / n}
$$

$\boldsymbol{Y}(k)$ is the spectrum obtained by Fourier transform of the signal. It is said that the specific DFT algorithm described in detail in many literatures (Oppenhiem et $a l, 1997)$. However, it is omitted in this paper. The amplitude and phase of each frequency could be calculated from the corresponding signal spectrum. Finally, the frequency response of the system could be estimated.

\section{Implementation of Frequency Response Estimation Toolbox and Application Examples}

\subsection{Introduction for the toolbox}

Currently the proposed-method based frequency response estimation toolbox has been developed and integrated into MWorks.Sysplorer platform.

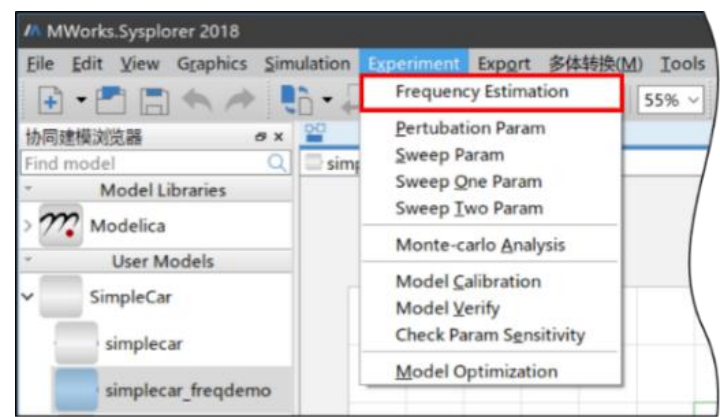

Figure 4 . Toolbox launch position

Main functions of the frequency response estimation toolbox are made up of creation of test signals, setting of linear input and output points along with graphical representation of the results of frequency response estimation. 
The creation of test signals is a highlight of the toolbox that could create Sinestream signal flexibly via UI interface.

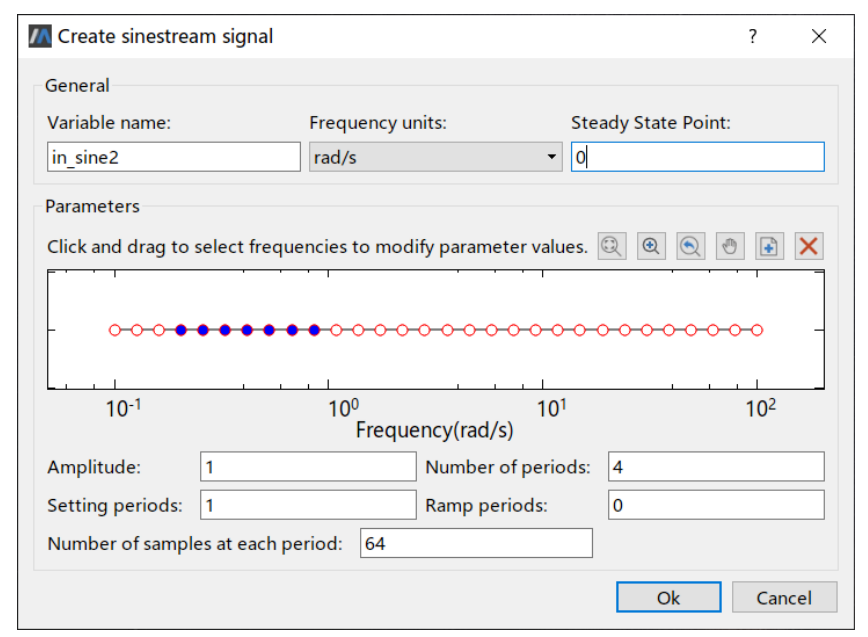

Figure 5. The create sine-stream input dialog box

As can be seen in Figure 5, Sinestream signal could be created visually by customizing system steady-state point, range of frequencies along with point numbers for estimation during it(logarithmically/linearly). In addition, customized parameters of signals for each frequency are as follows.
i. Amplitude;
ii. Number of periods;
iii. Settling periods;
iv. Ramp periods;
v. Number of samples at each period.

The estimation results of the system model can be presented in the toolbox in spectral data or Bode diagram.

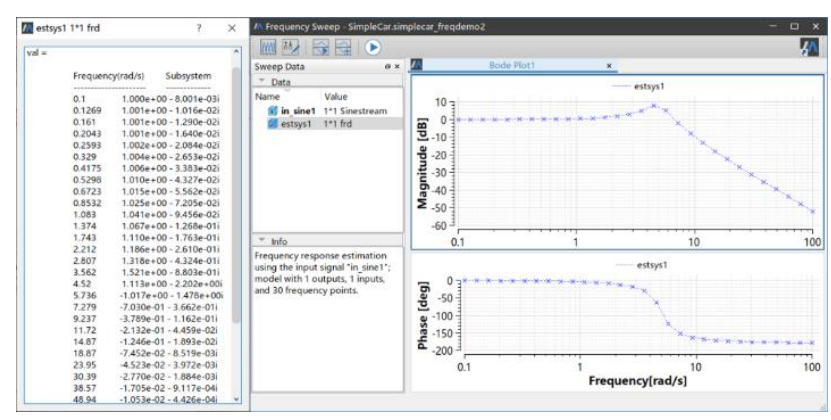

Figure 6. The results of estimation

Meanwhile, toolbox supports that obtaining the characteristics such as bandwidth of the system, resonance peak, amplitude margin and phase margin from estimation results.

\subsection{Application of A Simple Example}

Building model of a typical spring-damping-mass system of which parameters is shown as below in MWorks.Sysplorer. The model can be seen in Figure 7.

$$
M_{1}=6000 \mathrm{~kg}
$$

$$
\begin{aligned}
& M_{2}=160 \mathrm{~kg} \\
& k_{1}=5000 \mathrm{~N} / \mathrm{m} \\
& k_{2}=10000 \mathrm{~N} / \mathrm{m} \\
& D=1000 \mathrm{~N} /(\mathrm{m} / \mathrm{s})
\end{aligned}
$$

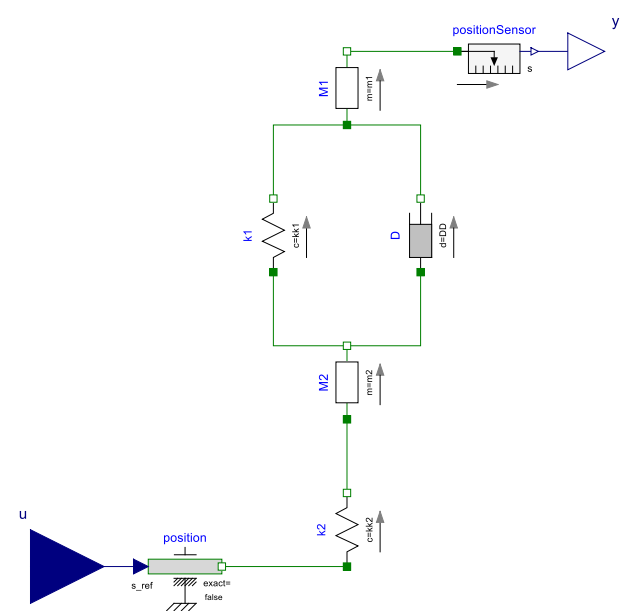

Figure 7. Spring-Damping-Mass system model

Estimating the frequency response of the system in the range of $[0.1,100] \mathrm{rad} / \mathrm{s}$ with frequency response estimation toolbox.

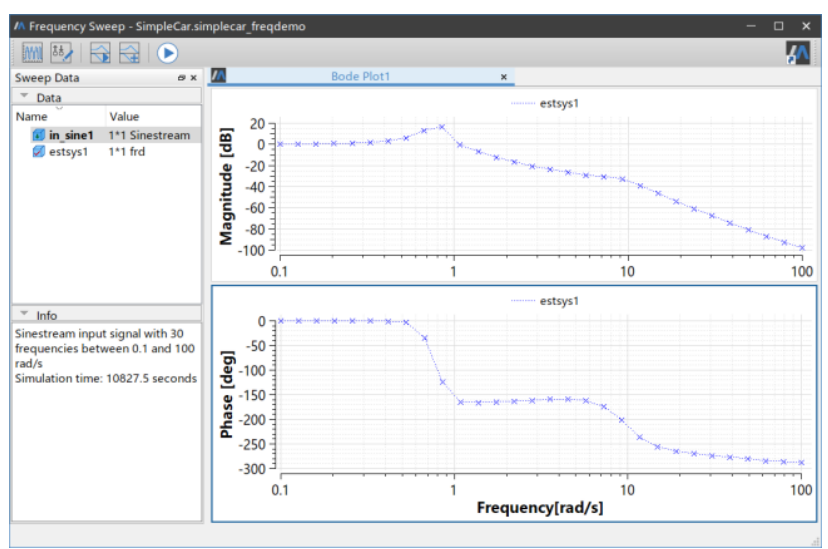

Figure 8. Estimation results of frequency response

The system of this example is actually a linear system. Therefore, the transfer function can be derived and then calculate the theoretical frequency response of the system to verify the accuracy of the above estimation results.

The transfer function of the system can be derived as follows:

$$
G(s)=\frac{10 s+50}{0.96 s^{4}+6.16 s^{3}+90.8 s^{2}+10 s+50}
$$

The theoretical frequency response of the system is calculated by MATLAB and compared with the results of estimation.

As shown in Figure 9, the result estimated by the proposed method is highly matched with the theoretical analytical result, which verifies the effectiveness of the proposed method and toolbox. 


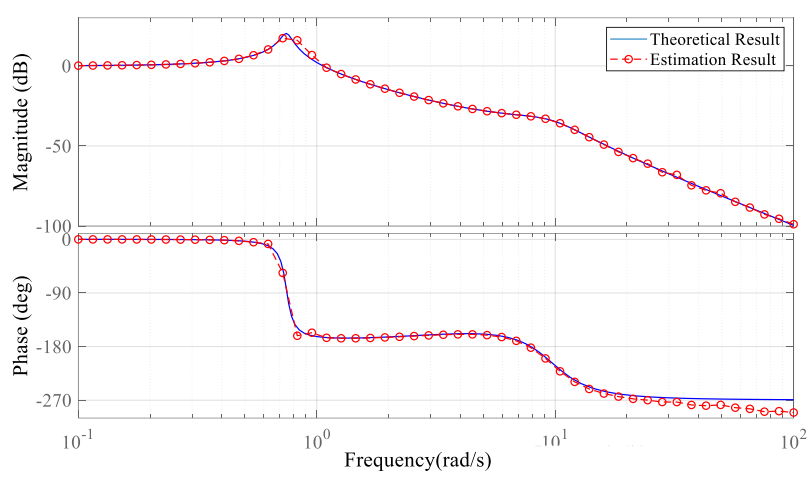

Figure 9.Verification of frequency response estimation

\subsection{Frequency Response Analysis of Electro- Hydraulic Actuator}

Taking the electro-hydraulic actuator of a certain type of aircraft flight control system as the research object, the actuator in the aircraft is usually called Power Control Unit (PCU). The design requirements of PCU includes the frequency response index. According to the principle of this type of PCU, its Modelica model is established as the following figure:

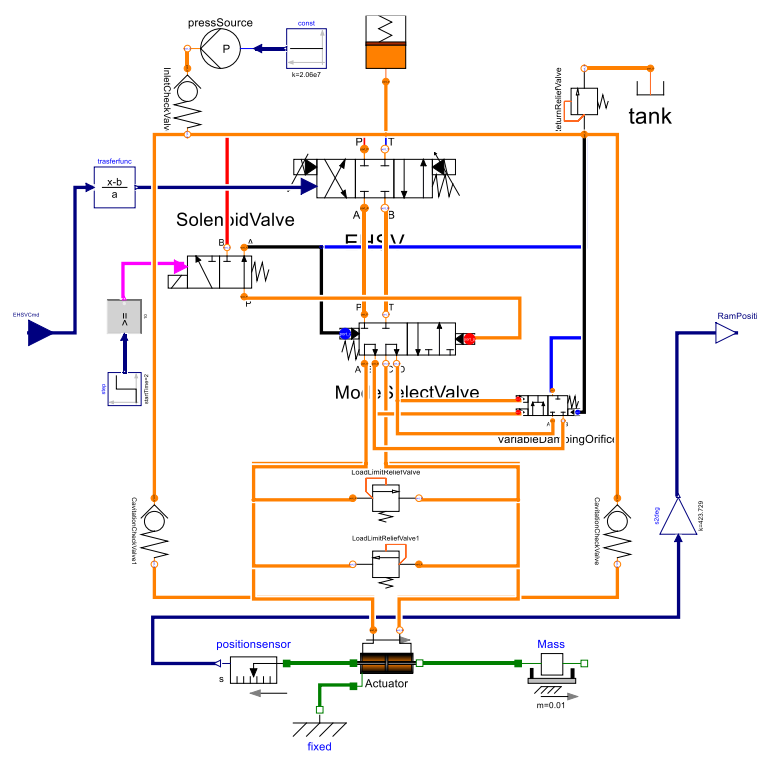

Figure 10. PCU model

Firstly, linearized function referred as linearizeModel on Dymola is applied to the PCU model which provide linearization process. The linearization result is shown as follows.

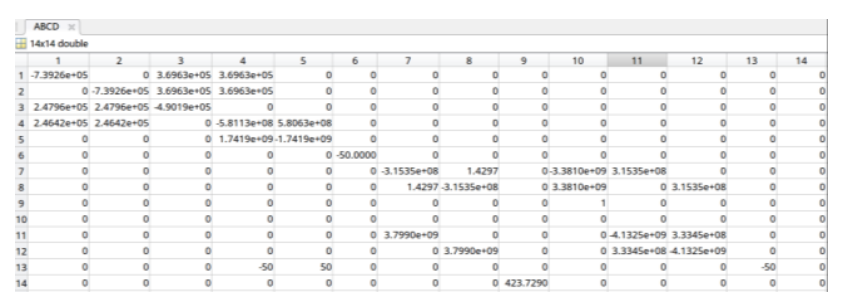

Figure 11. Linearization results of PCU model
As is shown in Figure 11, the thirteenth-order linear state-space model of the system could be obtained after linearization. Matrix $\boldsymbol{B}$ and $\boldsymbol{D}$ are as follows.

$$
\boldsymbol{B}=\left[\begin{array}{llll}
\overbrace{0} & 0 & \cdots & 0
\end{array}\right]^{T}, \quad \boldsymbol{D}=0
$$

It can be concluded that input of the system has no influence on state variables and system output. Therefore, it is incorrect to linearize the PCU model as many hydraulic components are contained of which strong nonlinearity could not meet the linearization conditions.

The frequency response is estimated in the frequency range of $[0.1,20] \mathrm{Hz}$ given the same conditions as those of frequency identification test of this type of PCU. The estimation result can be seen in the following figure.

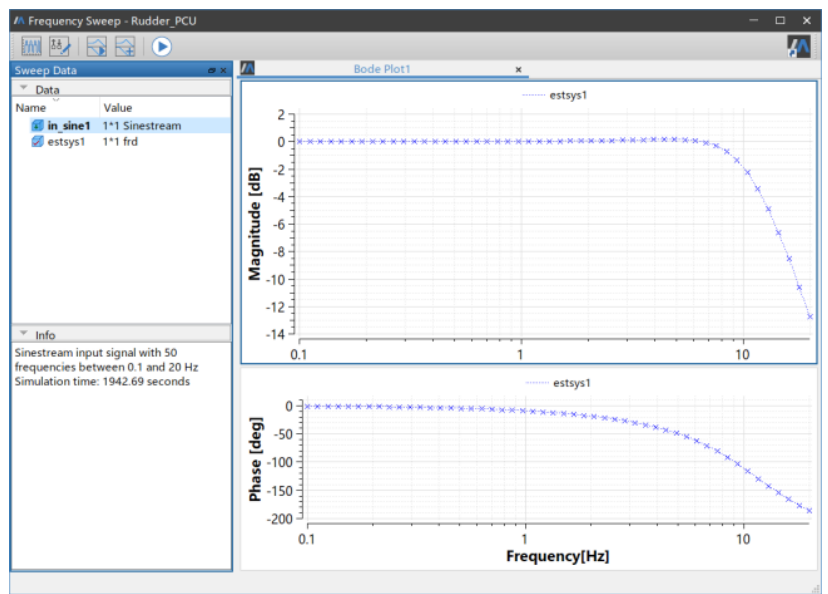

Figure 12. Estimation Results Of The PCU Model

Comparison between result of the frequency response estimation and result of the actual system frequency identification test is shown in Figure 13.

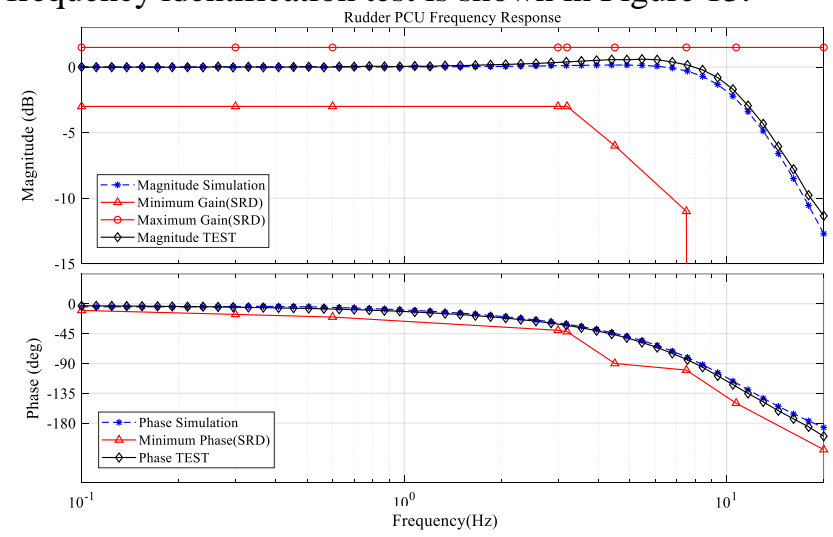

Figure 14. Verification of estimation results of PCU

As can be seen in Figure 14, red curve is represented as the frequency response range of the PCU defined in the Systems Requirements Document (SRD) while black curve is represented as the results of the frequency identification test. As for blue curve, it is defined as the results of estimation based on frequency response estimation toolbox. In summary, results of 
estimation confirms to those of identification test, thereby the validity and accuracy of the proposed method and corresponding toolbox.

\section{Conclusion}

Considering that the system has the characteristic of strong nonlinearity in the actual engineering, a method for estimating the frequency response of multi-domain Modelica model is proposed to copy with difficulty. Generating appropriate signal to excite system model and then estimating the system frequency response counting on output data from time-domain simulation to avoid stringent requirements for applying linearization method on system model. It is worth mentioning that frequency response estimation toolbox for Modelica model has been integrated into MWorks.Sysplorer platform to improve usability along with flexibility of the method. Last but not least, the viability of proposed method and toolbox could be verified by way of taking simulation above for example.

Currently the frequency response estimation introduced is applicable to single input multiple output (SIMO) Modelica model. There is high potential that this method could be widely used for multiple input multiple output (MIMO) system and finalizing promotion is established as target of next work. Besides, analysis on the method is mainly introduced, which is foundation for control system design subsequently. It will be important further work that guaranteeing availability of complete process of designing control system for Modelica model.

\section{References}

Andreas Abel, Tobias Nähring, Frequency-Domain Analysis Methods for Modelica Models, Proceedings of the 6th International Modelica Conference, 2008.

Alan V.Oppenhiem, Alan S. Willsky, With S. Hamid Nawab, Signals and Systems, Second Edition, Pearson, 1997.

Fritzson. Peter. Principles of object-oriented modeling and simulation with Modelica 2.1. John Wiley \& Sons, 2010.

Gene F. Franklin, J. David Powell, Abbas Emami-Naeini, Feedback Control of Dynamic Systems, Fifth Edition. Pearson Education US. 2009.

Garron Fish, Mike Dempsey, Juan Gabriel Delgado, Neil Roberts, Natural frequency analysis of Modelica powertrain models, Proceedings of the 9th International Modelica Conference, 2012.

http://www.fftw.org/

Katsuhiko Ogata. Modern Control Engineering, Fifth Edition. Pearson. 2010.

Loig Allain, Stéphane Neyrat, Antoine Viel, Linear Analysis Approach for Modelica Models, Proceedings of the 7th International Modelica Conference, 2009.

Marcus Baur, Martin Otter, Bernhard Thiele, Modelica Libraries for Linear Control Systems, Proceedings of the 7th International Modelica Conference, 2009.
Martin Otter, The LinearSystems library for continuous and discrete control systems, Proceedings of the 5th International Modelica Conference, 2006.

Richard C.Dorf, Robert H.Bishop. Modern Control Systems, 12th Edition. Pearson. 2016.

Tilman Bünte, Recording of Model Frequency Responses and Describing Functions in Modelica, Proceedings of the 8th International Modelica Conference, 2011.

The MathWorks Inc. https://ww2.mathworks.cn/help/ slcontrol/ug/frest.chirp.html 